\title{
Reducing the Complexity of the Multilayer Perceptron Network using the Loading Matrix
}

\author{
Mohamed Loay Dahhan \\ $\mathrm{PhD}$ Student \\ Dept. Statistics and Information Systems \\ Faculty of Economics \\ University of Aleppo, Syria
}

\author{
Yasser Almoussa \\ Professor \\ Dept. Statistics and Information Systems \\ Faculty of Economics \\ University of Aleppo, Syria
}

\begin{abstract}
In this paper, Researchers present three models of a Multilayer Perceptron Network (MLPs) based on the Factor Analysis with Principal Components method (PC) to reduce the degree of complexity of the neural network. In the first model, a neural network was built with all the variables in the input layer. In the second model, the results of the FA were adopted instead of the basic variables in the input layer, and in the third model, the Loading matrix was used to determine the number of nodes in the hidden layer and the weights that are associated with the input layer. Then compared the results of the models by determining the number of network weights that reflect the complexity of the network, in addition to the time of building and training the model and the accuracy of classification. The results of applying the models to a hypothetical database for the purposes of scientific research titled Bank Marketing showed that the model that inserted the factors in the hidden layer and preserved the high loading factors only is the best model in terms of low degree of complexity and maintaining classification accuracy.
\end{abstract}

\section{General Terms}

Complexity, Artificial Neural Networks ANN, Multivariate Analysis.

\section{Keywords}

Multilayer Perceptron Network MLP, Factor Analysis FA, Principle Component Analysis PCA, Complexity, Loading Matrix

\section{INTRODUCTION}

Artificial neural network (ANN) is a kind of cross-subject, which combines with Brain Science, Neuroscience, Cognitive Science, Psychology, Computer Science, and Mathematics [1]. There are many successful real world applications using ANN such as industry, business, science and classification problems [2-3]. Example of applications that have implemented using ANN are bankruptcy prediction [4], handwriting recognition [5], Time Series Forecasting [6]. Neural network has been more than 70 years until now; during these years, hundreds of network algorithm models have been proposed [7], and back-propagation (BP) neural network is one of the most mature and most widespread algorithms [8]. ANN with different structure gives different output for the same data set. Therefore, architectural design of ANN is very crucial and may be defined as an optimization problem [9]. MLP structure has three types of layers, the number of processing units (neurons) at input and output layer are fixed while these vary at the hidden layers. There is no straightforward criterion to calculate number of neurons in hidden layers nor is there any supporting theory for calculating the hidden layers. These architectural attributes are involved in the performance of ANN because a network with few layers and few neurons can cause under fitting while a large network easily leads to overfitting. [10]

Plenty of algorithms have been proposed for optimizing training rule as well as ANN architecture, which can suggest an optimal, or nearest optimal ANN structure but problem still needs further exploration. Many papers have used Multivariate Analysis (Principle Component Analysis (PCA), Factor Analysis (FA), Cluster Analysis (CA) ...) to improve ANN Architectures. Gross and Luttermann first proposed a hybrid architecture combining PCA with a multilayer perceptron for recognizing human faces [11]; after that many papers applied the Principle Component in Neural Network in many sectors, to predict software faults during testing [12], prediction and data analysis applications in meteorology and oceanography [13]. Another paper used an ANN for classification and a PCA in the preprocessing stage to classify the quality of food products by a feedforward ANN with one hidden layer and the backpropagation algorithm. The PCA as a feature extractor was applied to spectral data before training the ANN to reduce the amount of redundant information [14]. Bucinski et al. combined PCA and ANN in medicine using backpropagation ANN to classify patients into two categories, and PCA to extract some important features predictive for patients' survival [15]. Song et al. analyzed the high-spectral remote sensing data. By the integration of factor analysis (FA) and $\mathrm{NN}$ [16]. Zhang used integrating principal component analysis (PCA) with neural network in clinical diagnosis, diagnosed the swelling through measuring the amount of nucleoside in urine [17]. Gopi Combined independent component analysis (ICA) and NN to extract the features of forged images, to identify the forged images through NN [18]. Sousa et al used PCA analysis with varimax rotation to extract factors using ozone concentrations data. The results showed that principal components as inputs improved MLR and ANN models' prediction by eliminating data collinearity and the number of predictor variables [19]. Ravi and Pramodh placed principal components instead of the hidden layer of a multilayer perceptron (MLP) network. They proposed a new principal component neural network (PCNN) architecture to solve bankruptcy prediction problem in commercial banks. They replaced the hidden layer of an ANN by a 'principal component layer', which consists of a few selected principal components that perform the function of hidden nodes. The advantage of such architecture is that it reduces the number of weights by eliminating connections between the input layer and the principal component layer [20]; Miguel used tied hidden Factors to model speaker and session variability and a MAP adaptation of some of the parameters of the model [21]. Ding proposed a back-propagation (BP) neural network algorithm based on factor analysis (FA) and cluster analysis (CA), which is combined with the principles of FA and CA, 
and the architecture of BP neural network. The new algorithm reduces the feature dimensionality of the initial data through FA to simplify the network architecture [22]; Zekić Combined Two nonparametric methods to model entrepreneurial intentions: principal component analysis (PCA) and artificial neural networks (ANNs), it reveals benefits from the combination of the PCA and ANNs in modeling entrepreneurial intentions [23]

The main goal of this paper is to reduce the complexity degree of MLPs through reduction the number of connections (weights) between layers depending on the loading matrix that derived from factor analysis FA based on principal components analysis method. By building three models with different nodes and connections between layers, each model works only for single hidden layer. Then will compare the models based on confusion matrix and the needed time to build \& train the model. This paper is organized as follows. In Section 2 presents neural networks and loading matrix. Section 3 discusses Proposed models. Section 4 presents an experiment to show the accuracy of models. Conclusions are presented in Section 5.

\section{NEURAL NETWORKS \& LOADING MATRIX}

\subsection{Neural Network Architecture}

An artificial neural network, or simply neural network, is a type of artificial intelligence that attempts to mimic the way the human brain processes and stores information. It works by creating connections between mathematical processing elements, called neurons. Knowledge is encoded into the network through the strength of the connections between different neurons, called weights, and by creating groups, or layers, of neurons that work in parallel [24]. The system learns through a process of determining the number of neurons or nodes and adjusting the weights for the connections based upon training data. The architecture consists of not only input and output layer but also one or more intermediary layers called hidden layers. The computation units of the hidden layer called as hidden neurons. The function of hidden neurons is to intervene between the external input and the network output in some useful manner [25]. The performance of it fully depends upon the selection of architecture and training algorithm. MLP architecture can be tweaked using several parameters, such as the number of hidden layers, number of hidden neurons at each hidden layer and number of connections between layers [9].

The most widely used architecture in ANNs has been the Multiple Layer Perceptron (MLP), trained with the Back Propagation (BP) error-learning algorithm [26]. In this architecture, nodes are partitioned into layers numbered 0 to $\mathrm{L}$, where the layer number indicates the distance of a node from the input nodes. The lowermost layer is the input layer numbered as layer 0 , and the topmost layer is the output layer numbered as layer L. layers numbered between 0 and $\mathrm{L}$ are hidden layers [27-28-29], The $\omega$ number of weights and biases of the NN can be seen in Eq. (1) [30].

$\omega=(N i+1) N h+(N h+1) N h * N l+(N h+1) N o$

Where $\mathrm{N}_{\mathrm{I}}, \mathrm{N}_{\mathrm{h}}$, and $\mathrm{N}_{\mathrm{O}}$ are the numbers of the nodes in the input layer, the hidden layer, and the output layer. $\mathrm{N}_{\mathrm{l}}$ is the number of hidden layers as presented in Fig. 1.

In classification problems, the most common activation function is Softmax. Because the output vector has a component corresponding to each target class, and the softmax output is interpreted as the probability of the input belonging to the corresponding class.

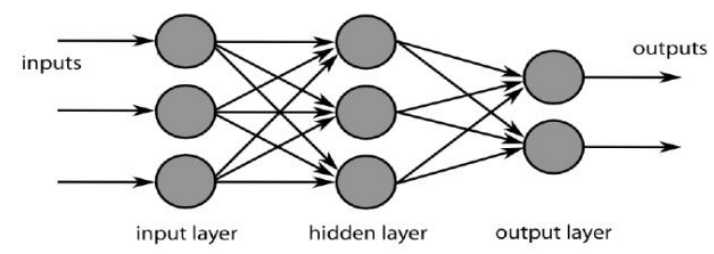

Figure 1: Neural Network Architecture

The softmax function differs from sigmoid functions in that it does not operate element-wise on a vector. Rather, the softmax function applies to an entire vector. If $x=$ $[x 1, x 2, \ldots, x n], \quad$ then $\quad$ its softmax $\mu(x)=[\mu(x 1), \mu(x 2), \ldots, \mu(x n)]$, where $[31]:$

$$
\mu\left(x_{i}\right)=\frac{e^{x_{i}}}{\sum_{j} e^{x_{j}}}
$$

The backpropagation algorithm is a generalization of the least mean squared algorithm that modifies network weights to minimize the mean squared error between the desired and actual outputs of the network. Backpropagation uses supervised learning in which the network is trained using data for which inputs as well as desired outputs are known. Backpropagation is a simple training method that uses the calculated gradients of a neural network to adjust the weights of the neural network. As these weights are adjusted the neural network should produce more desirable output. The global error of the neural network should fall as it is trained [27-32].

ANN model trained by Cross-Validation Method, in k-fold cross-validation, the available learning set is partitioned into $\mathrm{k}$ disjoint subsets of approximately equal size. Fold refers to the number of resulting subsets. This partitioning is performed by randomly sampling cases from the learning set without replacement. The model is trained using $\mathrm{k}-1$ subsets, which, together, represent the training set. Then, the model is applied to the remaining subset, which is denoted as the validation set, and the performance is measured. This procedure is repeated until each of the $\mathrm{k}$ subsets has served as validation set. The average of the $\mathrm{k}$ performance measurements on the $\mathrm{k}$ validation sets is the cross-validated performance [33].

\subsection{MLP Complexity degree}

Complexity degree of Multi-Layer Perceptron could be estimated by the needed time to train and generalize the desired model. The essential storage requirement for the BP algorithm consists of all the $\mathrm{N}_{\mathrm{w}}$ weights of the network. The computational complexity per iteration of the BP is around $\mathrm{N}_{\mathrm{w}}$ multiplications for the forward pass, around $2 \mathrm{~N}_{\mathrm{w}}$ multiplications for the backward pass [34]. The process of presenting all the examples in the pattern set, with each example being presented once, is called an epoch. Neural networks are trained by presenting all the examples cyclically by epoch, until the convergence criteria is reached. The training examples should be presented to the network in a random order during each epoch [35].

Complexity degree of a Multi-Layer Perceptron is determined by the number of free parameters that it has (weights and biases), which in turn is determined by the number of neurons [36]. According to $\mathrm{Eq}(1)$ number of weights calculates by the sum of weights between each layer on sequence, as it 
clarified:

$$
\omega=\sum_{i=0}^{i=L-1}\left(\mathrm{\eta}_{i}+b_{i}\right) * \mathrm{\eta}_{(i+1)}
$$

- $\quad \omega$ : Total number of weights.

- $\mathrm{\eta}_{i}$ : Number of nodes in the layer i.

- $\quad b_{i}$ : Bias node in the layer i.

- $\quad L:$ Number of layers.

\subsection{Factor Analysis and Loading Matrix}

The essential purpose of factor analysis FA is to describe, if possible, the covariance relationships among many variables in terms of a few underlying, but unobservable, random quantities called Factors. Factor analysis can be considered an extension of principal component analysis PCA. Both can be viewed as attempts to approximate the covariance matrix. The approximation based on factor analysis model is more elaborate [37]. Despite similarities, there is a fundamental difference between them: PCA is a linear combination of variables; FA is a measurement model of a latent variable. The common estimation method of factors is principle factors, used the principal component method.

The observable random vector $\boldsymbol{X}$, with $\boldsymbol{p}$ components has mean $\mu$ and covariance matrix $\Sigma$. The factor model postulates that $\boldsymbol{X}$ is linearly dependent upon a few un observable random values $F_{1}, F_{2}, \ldots, F_{m}$ called Common factors, and $p$ additional sources of variation $\square 1, \square 2, \ldots ., \square p$, called errors or sometimes called specific factors. The factor analysis model is:

$$
\begin{gathered}
X_{1}-\vartheta_{1}=\ell_{11} F_{1}+\ell_{12} F_{2}+\ldots+\ell_{1 m} F_{m}+\varepsilon_{1} \\
X_{2}-\vartheta_{2}=\ell_{21} F_{1}+\ell_{22} F_{2}+\ldots+\ell_{2 m} F_{m}+\varepsilon_{2} \\
\vdots \\
X_{p}-Q_{p}=\ell_{p 1} F_{1}+\ell_{p 2} F_{2}+\ldots+\ell_{p m} F_{m}+\varepsilon_{p}
\end{gathered}
$$

- $\nabla_{i}:$ mean of variable $\mathrm{i}$.

- $\varepsilon_{i}:$ i th specific factor.

- $\quad F_{j: \mathrm{j}} \mathrm{j}$ the common factor.

- $\quad \ell_{i j}$ : Loading of the $\mathrm{i}_{\mathrm{th}}$ on the $\mathrm{j}_{\mathrm{th}}$ factor.

Or, in matrix notation,

$$
X-\mu_{(p * 1)}=L_{(p * m)} * F_{(m * 1)}+\varepsilon_{(p * 1)}
$$

Therefore, $L$ is the matrix of factor loadings [37]. One widely used method to determine factors number is to let the number of factors equal the number of eigenvalues of the sample correlation matrix that are greater than 1. It is called KaiserGuttman criterion. Initial solution of factor loadings should be rotated to find a simple structure. Several methods for rotation to a simple structure have been proposed. The rotational methods are classified as orthogonal and oblique rotations, among the orthogonal rotations; Kaiser's vari-max rotation is by far the most frequently used. Each factor keeps variables whose coefficient of loading is greater than 0.5 [38].

\section{PROPOSED MODELS}

This paper supposes a neural network with three layers (input layer- hidden layer- output layer) to classify a binary variable with two classes, and:
- $\quad m$ : number of nodes in the input layer.

- $\quad h$ : number of nodes in the hidden layer.

- $\quad o$ : number of nodes in the output layer, its value is two.

In addition, there is a bias node in both input layer and hidden layer. According to $\mathrm{Eq}(2)$ the complexity degree (number of weights) can be calculated as follows:

$\omega=(m+1) * h+(h+1) * 2$

- $\quad(m+1) * h$ : represent the number of connections between input layer and hidden layer.

$(h+1) * 2$ : represent the number of connections between hidden layer and output layer.

Then:

$\omega=(m * h+h)+(2 h+2)$

The number of nodes in hidden layer depends on trial and error concept. With too few nodes, the network may not be powerful enough for a given learning task. With a large number of nodes, computation is too expensive.

In this paper, according to Rule of thumb method researchers suppose the nodes in hidden layer is the average of the nodes number between input and output layers, so $h=\frac{m+2}{2}$ [39]; Then the number of weights in the model as following:

$$
\begin{gathered}
\omega=\left((m+1) *\left(\frac{m+2}{2}\right)\right)+2\left(\frac{m+2}{2}+1\right) \\
\omega=\left(m * \frac{m+2}{2}+\frac{m+2}{2}\right)+\left(2 \frac{m+2}{2}+2\right) \\
\omega=\frac{m^{2}}{2}+m+\frac{m}{2}+1+m+2+2 \\
\omega=\frac{m^{2}}{2}+\frac{5 m}{2}+5
\end{gathered}
$$

In this paper, three different models of the neural network were studied, to evaluate the models researchers depended on the following measures:

1. Time to build and train the model.

2. Valid classification percentage.

\subsection{First model (ANN)}

Multi-layers neural network without any modification on the network architecture; the number of weights given as follows:

$$
\omega 1=\frac{m^{2}}{2}+\frac{5 m}{2}+5
$$

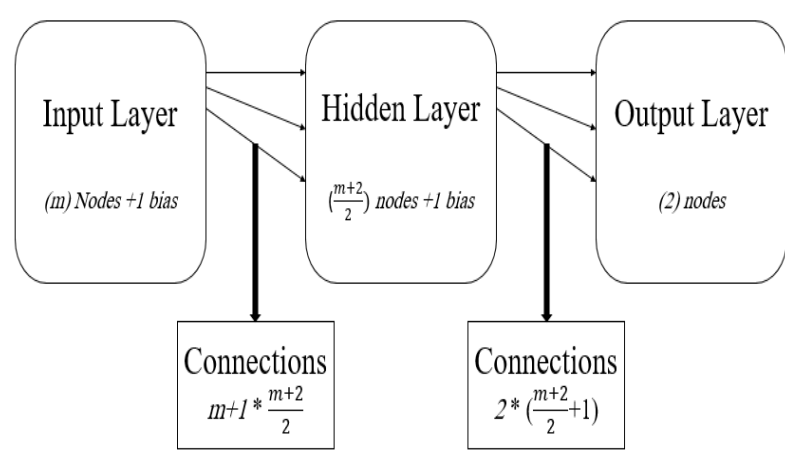

Figure 3. Architecture of Model 1 


\subsection{Second Model: (ANN with FA in Input Layer)}

We applied factor analysis FA with principal component analysis PCA method; the factors $p$ was merging into the neural network architecture by replacing the input layer that had $m$ node with the factors $p$. and:

$$
1 \leq p \ll m
$$

Then the number of weights given as following:

$$
\omega 2=\frac{p^{2}}{2}+\frac{5 p}{2}+5
$$

The complexity degree (number of weights) of the second model are always lower than complexity degree (number of weights) of the first model because of expression (9). Then the second model needs a lower training time:

$$
\omega 2 \ll \omega 1
$$

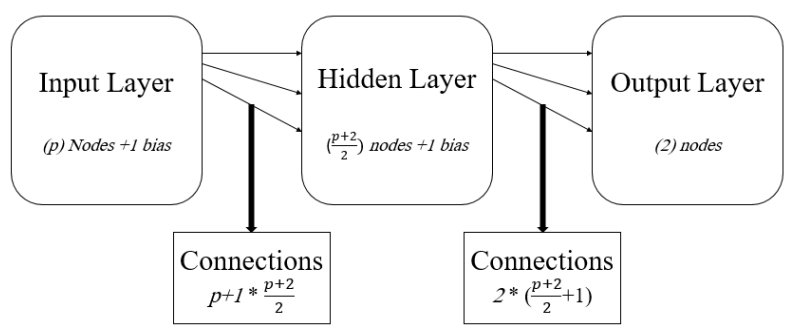

Figure 4. Architecture of Model 2

\subsection{Third Model: (ANN with FA in Hidden Layer)}

We also applied FA with PCA method. Then merge previous analysis into the neural network architecture by identifying the hidden layer nodes based on the factors $p$ and the weights that linking it with the input layer nodes, which produced from the loading matrix $\ell_{i j}>0.5$. The number of nodes in input layer could be lower than $m$ by $\alpha$, which represent the number of unloaded variables on any factor $p$. The number of connections between input layer nodes and hidden layer nodes is $\beta$, which represent the loaded variables on factors and bigger than 0.5 , considering validity of $m^{2}>>\beta$. Then the complexity degree (number of weights) given as following expression:

$$
\begin{aligned}
& \omega 3=\beta+(p+1) * 2 \\
& \omega 3=\beta+2 p+2
\end{aligned}
$$

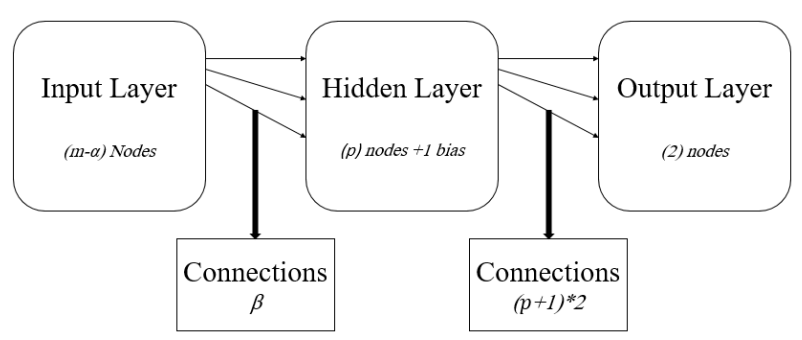

Figure 4. Architecture of Model 3

In addition, considering that:

$$
\begin{gathered}
p<m \\
\beta \ll m^{2}
\end{gathered}
$$

Then:

$$
\omega 3<\omega 2<\omega 1
$$

\section{PRACTICAL EXPERIMENT}

This study applied on a bank-marketing database [40] created

\begin{tabular}{|c|c|}
\hline \multicolumn{2}{|r|}{ Numeric Variables } \\
\hline Code & Description \\
\hline $\mathrm{X} 1$ & Age \\
\hline $\mathrm{X} 11$ & Last contact duration, in seconds. \\
\hline $\mathrm{X} 12$ & $\begin{array}{l}\text { number of contacts performed during this } \\
\text { campaign and for this client }\end{array}$ \\
\hline $\mathrm{X} 13$ & $\begin{array}{l}\text { number of days that passed by after the client } \\
\text { was last contacted from a previous campaign }\end{array}$ \\
\hline $\mathrm{X} 14$ & $\begin{array}{l}\text { number of contacts performed before this } \\
\text { campaign and for this client }\end{array}$ \\
\hline $\mathrm{X} 16$ & employment variation rate - quarterly indicator \\
\hline $\mathrm{X} 17$ & consumer price index - monthly indicator \\
\hline $\mathrm{X} 18$ & consumer confidence index - monthly indicator \\
\hline $\mathrm{X} 19$ & euribor 3 month rate - daily indicator \\
\hline $\mathrm{X} 20$ & number of employees - quarterly indicator \\
\hline \multicolumn{2}{|r|}{ Nominal variables ( $0=$ ="No", 1="yes") } \\
\hline $\mathrm{X} 5$ & Has credit in default? \\
\hline $\mathrm{X} 6$ & Has housing loan? \\
\hline $\mathrm{X} 7$ & Has personal loan? \\
\hline $\mathrm{Y}$ & Has the client subscribed a term deposit? \\
\hline \multicolumn{2}{|r|}{ Categorical Variables ( 1 for the $1^{\text {st }}$ category $\ldots$ ) } \\
\hline $\mathrm{X} 2$ & type of job \\
\hline $\mathrm{X} 3$ & marital status \\
\hline $\mathrm{X} 4$ & Education Level \\
\hline $\mathrm{X} 8$ & contact communication type \\
\hline $\mathrm{X} 9$ & last contact month of year \\
\hline $\mathrm{X} 10$ & last contact day of the week \\
\hline $\mathrm{X} 15$ & outcome of the previous marketing campaign \\
\hline
\end{tabular}
to predict the success of marketing campaigns were based on phone calls. The number of studied cases was 41188, the missing data recoded with the "unknown" label for the categorical variables, series average for the numeric variables.

The attributes divided into 21 variables explained in the following table:

Table.1. Variables Dictionary

\subsection{FA with PCA result:}

FA was applied on the variables (X1, X2 ...X20), considering $\mathrm{Y}$ is the target variable. By using SPSS the result as following:

Multi-Collinearity: the value of determinant is $(0.00007 \neq 0)$ which means factor analysis can be applied on the studied dataset, and then none of variables need to be deleted. 
KMO Test: As in Table.2, the value of KMO is 0.543 , which is bigger than 0.5 . Then the sample size is good enough to apply the analysis.

Bartlett's Test: from the table 2, the significant level is 0.000 . Therefore, researchers refuse the null hypothesis that the correlation matrix is an identity matrix.

Table.2 KMO \& Bartlett's Test Results

\begin{tabular}{|l|l|}
\hline KMO Value & 0.543 \\
\hline Bartlett (sig) & 0.000 \\
\hline
\end{tabular}

Communalities: the default value of communalities is (1) based on PCA method and Correlation matrix. The value of communalities refer to the percentage of variable variances explained by the common factors. For example, the value of variable X15 is 0.964 ; refer to $96.4 \%$ of the variable X15 variances was explained by common factors.

In general most of common factors values explain a good percentage of variable variances, and the less percentage was for X4 (0.486). As cleared in Table.3.

Table 3 Communalities

\begin{tabular}{|c|c|c|c|c|c|}
\hline $\begin{array}{c}\text { Variabl } \\
\mathbf{e}\end{array}$ & $\begin{array}{c}\text { defaul } \\
\mathbf{t}\end{array}$ & $\begin{array}{c}\text { Selecte } \\
\mathbf{d}\end{array}$ & $\begin{array}{c}\text { Variabl } \\
\mathbf{e}\end{array}$ & $\begin{array}{c}\text { defaul } \\
\mathbf{t}\end{array}$ & $\begin{array}{c}\text { Selecte } \\
\mathbf{d}\end{array}$ \\
\hline $\mathrm{X} 1$ & 1 & .688 & $\mathrm{X} 11$ & 1 & .560 \\
\hline $\mathrm{X} 2$ & 1 & .536 & $\mathrm{X} 12$ & 1 & .550 \\
\hline $\mathrm{X} 3$ & 1 & .569 & $\mathrm{X} 13$ & 1 & .920 \\
\hline $\mathrm{X} 4$ & 1 & .486 & $\mathrm{X} 14$ & 1 & .940 \\
\hline $\mathrm{X} 5$ & 1 & .290 & $\mathrm{X} 15$ & 1 & .964 \\
\hline $\mathrm{X} 6$ & 1 & .645 & $\mathrm{X} 16$ & 1 & .948 \\
\hline $\mathrm{X} 7$ & 1 & .654 & $\mathrm{X} 17$ & 1 & .800 \\
\hline $\mathrm{X} 8$ & 1 & .741 & $\mathrm{X} 18$ & 1 & .504 \\
\hline $\mathrm{X} 9$ & 1 & .808 & $\mathrm{X} 19$ & 1 & .976 \\
\hline $\mathrm{X} 10$ & 1 & .957 & $\mathrm{X} 20$ & 1 & .888 \\
\hline
\end{tabular}

Total Explained Variance: variables (X1, X2...X20) represented by nine factors. Based on Kaiser Rule, first nine factors was taken because of its Eigenvalues $(E V>1)$.

Table 4 Total Explained Variance

\begin{tabular}{|c|c|c|c|}
\hline & EV & Explained Variance \% & Cumulative \% \\
\hline F1 & 4.254 & $\mathbf{2 1 . 2 7 \%}$ & $21.27 \%$ \\
\hline F2 & 1.699 & $8.497 \%$ & $29.766 \%$ \\
\hline F3 & 1.556 & $7.778 \%$ & $37.545 \%$ \\
\hline F4 & 1.478 & $7.392 \%$ & $44.936 \%$ \\
\hline F5 & 1.286 & $6.432 \%$ & $51.368 \%$ \\
\hline F6 & 1.082 & $5.411 \%$ & $56.779 \%$ \\
\hline F7 & 1.042 & $5.21 \%$ & $61.989 \%$ \\
\hline F8 & 1.026 & $5.13 \%$ & $67.119 \%$ \\
\hline F9 & 1.001 & $5.003 \%$ & $\mathbf{7 2 . 1 2 2 \%}$ \\
\hline
\end{tabular}

As shown in table 4 the cumulative percent of explained variances is $72.122 \%$ of the total variance, and the first factor has the highest EV than the other factors 4.254 explain $21.27 \%$ of the total variances for the studied variables.

Loading matrix after rotation: It shows the correlation degree between variables and each factor of the selected factors using Varimax rotation. The variables with $\boldsymbol{\ell}_{i j \text { : }}<0.5$ was ignored

Results: Nine factors was derived from variables summarizes in the table 6 below:

Table 4 Factors Result

\begin{tabular}{|c|c|c|}
\hline Factor & Loaded variables & Variance explained \\
\hline F1 & $\mathrm{X} 8, \mathrm{X} 16, \mathrm{X} 17, \mathrm{X} 19, \mathrm{X} 20$ & $21.27 \%$ \\
\hline $\mathrm{F} 2$ & $\mathrm{X} 13, \mathrm{X} 14$ & $8.497 \%$ \\
\hline $\mathrm{F} 3$ & $\mathrm{X} 1, \mathrm{X} 3$ & $7.778 \%$ \\
\hline F4 & $\mathrm{X} 8, \mathrm{X} 9$ & $7.392 \%$ \\
\hline F5 & $\mathrm{X} 13, \mathrm{X} 15$ & $6.432 \%$ \\
\hline F6 & $\mathrm{X} 6, \mathrm{X} 7$ & $5.411 \%$ \\
\hline F7 & $\mathrm{X} 2, \mathrm{X} 4$ & $5.21 \%$ \\
\hline F8 & $\mathrm{X} 11, \mathrm{X} 12$ & $5.13 \%$ \\
\hline F9 & $\mathrm{X} 10$ & $5.003 \%$ \\
\hline
\end{tabular}

Then the representation of factor model formalized as next expressions:

$$
\begin{aligned}
& F_{1}=0.511 X_{8}+0.958 X_{16}+0.793 X_{17}+0.964 X_{19} \\
& +0.864 X_{20} \\
& F_{2}=0.708 X_{13}-0.887 X_{14} \\
& F_{3}=0.825 X_{1}-0.748 X_{3} \\
& F_{4}=0.859 X_{9}-0.664 X_{8} \\
& F_{5}=0.967 X_{15}-0.605 X_{13} \\
& F_{6}=0.799 X_{6}+0.806 X_{7} \\
& F_{7}=0.710 X_{2}-0.611 X_{4} \\
& F_{8}=0.712 X_{12}-0.726 X_{11} \\
& F_{9}=0.977 X_{10}
\end{aligned}
$$

Total explained variances was $72.122 \%$.Then we can depend on the proposed factors because total explained variances greater than $60 \%$.

In addition, two variables were ignored $(\mathrm{X} 5, \mathrm{X} 18)$ because there are not loaded on any factor.

\subsection{Applying Models:}

Multi-layer Perceptron architecture has a good fitting with proposed classification issue, the researchers determined the static attributes for each of the proposed models summarizes as following:

\section{Table 7 Neural Network static attributes}

\begin{tabular}{|c|c|}
\hline Total Number of Instances & 41188 \\
\hline Training and Testing Method & Cross-Validation \\
\hline Variables Scaling & Standard \\
\hline
\end{tabular}




\begin{tabular}{|c|c|}
\hline Optimization Algorithm & Softmax \\
\hline Seeds & 0 \\
\hline Learning Rate & $\eta=0.4$ \\
\hline Momentum & $\alpha=0.3$ \\
\hline Validation Threshold & 20 \\
\hline
\end{tabular}

\subsubsection{Model Architecture:}

First Model (ANN): The 20 variables represented in input layer and the target variable as in output layer, so the number of output layer nodes is two based on the variable form. Number of nodes in the hidden layer is $\frac{20+2}{2}=11$ node. Moreover, the number of weights is the connections between each node in input layer with the nodes in hidden layer, and each node in hidden layer connected with the nodes in output layer. Then number of connections or weights calculated as follows:

$$
\omega 1=\frac{20^{2}}{2}+\frac{5 * 20}{2}+5=255
$$

Second model (ANN with FA in input layer): Factors represented in input layer (variables are replaced by the derived factors as inputs in input layer). Therefore, the nodes number in input layer is 9 nodes, and the number of nodes in hidden layer is $\frac{9+2}{2} \cong 6$. The nodes number in output layer is 2 . Then number of the connections or weights calculated as follows:

$$
\omega 2=\frac{9^{2}}{2}+\frac{5 * 9}{2}+5=68
$$

Third model (ANN with FA in hidden layer): Input layer represented with total number of variables except (X5, X18), so number of nodes in input layer is 18 . And for the number of nodes in hidden layer determined based on factor analysis results by number of derived factors values that its EV greater than 1 which is $p=9$ nodes. Like the previous models the nodes of output layer number is 2 . Then for the weights number, it is calculated based on number of loaded variables on factors $\beta=20$ added to the number of connections between hidden and output layer $(p+1) * 2$ as following:

$$
\omega 3=20+(2 * 9+2)=40
$$

Table (8) clear the calculated components of neural networks architecture based on the discussed models above:

Table 8 Components of neural network architecture

\begin{tabular}{|c|c|c|c|}
\hline Model & ANN & $\begin{array}{c}\text { ANN with } \\
\text { FA in } \\
\text { input layer }\end{array}$ & $\begin{array}{c}\text { ANN with } \\
\text { FA in hidden } \\
\text { layer }\end{array}$ \\
\hline Input Layer & 20 Node & 9 Node & 18 Node \\
\hline Hidden Layer & 11 Node & 6 Node & 9 Node \\
\hline Output Layer & 2 Node & 2 Node & 2 Node \\
\hline Total Weights & 255 & 68 & 40 \\
\hline
\end{tabular}

\subsubsection{Confusion Matrix}

It used to describe the performance of a classification model on a set of test data for which the true values are known. The following tables clear the confusion matrix for each model.
ANN model: Model classified accuracy is $90.89 \%$. Number of true classified instances is 37436

Table 9 Confusion Matrix for ANN model

\begin{tabular}{|c|c|c|}
\hline \multirow{2}{*}{ Actual } & \multicolumn{2}{|c|}{ Classifier } \\
\cline { 2 - 3 } & $\mathrm{a}=\mathrm{F}$ & $\mathrm{b}=\mathrm{T}$ \\
\hline $\mathrm{a}=\mathrm{F}$ & 35120 & 1428 \\
\hline $\mathrm{b}=\mathrm{T}$ & 2324 & 2316 \\
\hline $\begin{array}{c}\text { True classified } \\
\text { percentage }\end{array}$ & $35120+2316 / 41188=90.89 \%$ \\
\hline
\end{tabular}

ANN with FA in input layer model: Model classified accuracy is $90.77 \%$. Number of true classified instances is 37389 .

Table 10 Confusion Matrix for ANN with FA in Input Layer model

\begin{tabular}{|c|c|c|}
\hline \multirow{2}{*}{ Actual } & $\mathrm{2}$ & \multicolumn{2}{|c|}{ Classifier } \\
\cline { 2 - 3 } & $\mathrm{a}=\mathrm{F}$ & $\mathrm{b}=\mathrm{T}$ \\
\hline $\mathrm{a}=\mathrm{F}$ & 35324 & 1224 \\
\hline $\mathrm{b}=\mathrm{T}$ & 2575 & 2065 \\
\hline $\begin{array}{c}\text { True classified } \\
\text { percentage }\end{array}$ & $35324+2065 / 41188=90.77 \%$ \\
\hline
\end{tabular}

ANN with FA in hidden layer model: Model classified accuracy is $90.85 \%$. Number of true classified instances is 37421.

Table 11 Confusion Matrix for ANN with FA in Hidden Layer model

\begin{tabular}{|c|c|c|}
\hline \multirow{2}{*}{ Actual } & \multicolumn{2}{|c|}{ Classifier } \\
\cline { 2 - 3 } & $\mathrm{a}=\mathrm{F}$ & $\mathrm{b}=\mathrm{T}$ \\
\hline $\mathrm{a}=\mathrm{F}$ & 35413 & 1135 \\
\hline $\mathrm{b}=\mathrm{T}$ & 2632 & 2008 \\
\hline $\begin{array}{c}\text { True classified } \\
\text { percentage }\end{array}$ & $35413+2008 / 41188=90.85 \%$ \\
\hline
\end{tabular}

\subsection{Discussion}

Table (12) reviews results comparison between three models. Classification Rate at the ANN Model was the greatest $90.89 \%$, then ANN with FA in hidden layer model and the lowest for the ANN with FA in input layer model. In addition, it is interesting to note that classification rate for the ANN with FA in hidden layer model was so close to the highest one with a low difference equal to $0.04 \%$. The highest value for kappa statistic was for the ANN model 0.5024. Whereas the lowest value for the ANN with FA in hidden layer model 0.4676 , with small different between them.

Time needed to build and train the first model was 1239 seconds, which is extremely high, and then the second model with 486 seconds for building and training and the lowest time was for the third model around 319 seconds. Finally notice that model sensitivity for the classifier $(b=T)$ could return to the origin of the proposed issue in dataset, we could predict to deposit subscription $(\mathrm{a}=\mathrm{F})$ because of quantity and diversity of 
the instances.

Table 12 Models implementation results summary

\begin{tabular}{|c|c|c|c|}
\hline Model & ANN & $\begin{array}{c}\text { ANN with } \\
\text { FA in } \\
\text { input layer }\end{array}$ & $\begin{array}{c}\text { ANN with } \\
\text { FA in } \\
\text { hidden layer }\end{array}$ \\
\hline $\begin{array}{c}\text { True classified } \\
\text { instances }\end{array}$ & 37436 & 37389 & 37421 \\
\hline $\begin{array}{c}\text { False classified } \\
\text { instances }\end{array}$ & 3752 & 3799 & 3767 \\
\hline $\begin{array}{c}\text { Kappa statistic } \\
\text { True classifier } \\
\text { percentage }\end{array}$ & 0.5024 & 0.4715 & 0.4676 \\
\hline $\begin{array}{c}\text { Model building \& } \\
\text { training time }\end{array}$ & $1239 \mathrm{sec}$ & $486 \mathrm{sec}$ & $319 \mathrm{sec}$ \\
\hline $\begin{array}{c}\text { Complexity } \\
\text { degree } \\
\text { (Weights number) }\end{array}$ & 255 & 68 & 40.854 \\
\hline
\end{tabular}

Fig (1) shows the results of the comparison between models, and clearly the superiority of the ANN with FA in hidden ANN

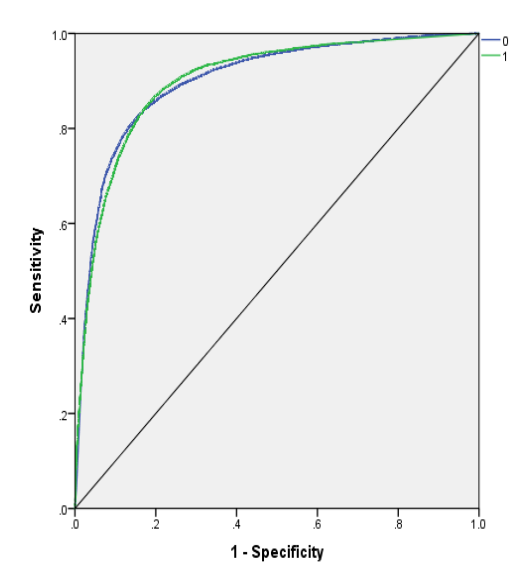

Area under the Curve: 0.920
ANN with FA in input layer

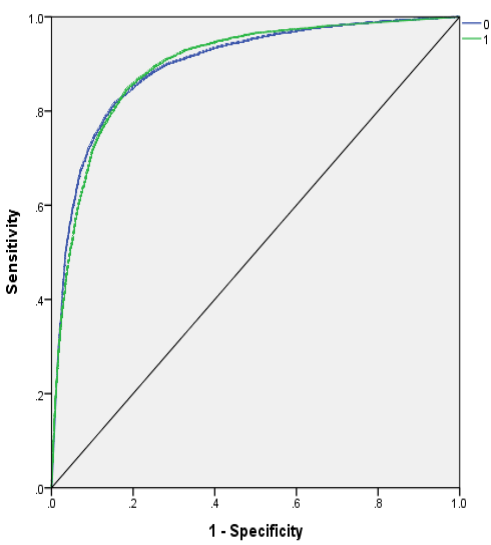

Area under the Curve: 0.898 layer model in complexity degree building \& training time, and its proximity to the best correct classification

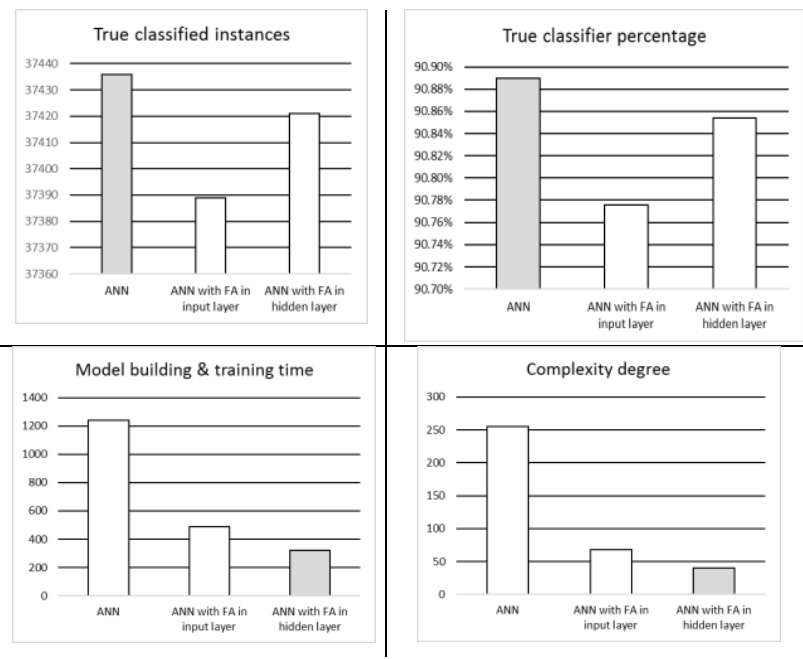

Fig 1: Comperation Charts between Models

Fig (2) shows ROC curve for each model, it is clear that the largest area under the curve for the ANN model, then ANN with FA in hidden layer model, and the lowest for the ANN with FA in input layer model. This is reflected in the accuracy of the True classifier percentage of these models.

\section{Fig 2: ROC Curves comparison of models}

\section{CONCLUSIONS}

1. Using multi-layer perceptron made a good performance with binary variable classification based on multi-dimension database.

2. When depending on factors in the input layer, some of variable had no connection with any node in the hidden layer, because this variable unloaded on any factor, whereas all variable was connected with hidden layer nodes without using factor analysis.

3. Replacing the original variables with factors in ANN with FA in input layer model has a good impact on reduction the complexity degree for the network, and a good classification accuracy comparing to the original model with less building and training time.

4. Reducing the complexity degree of ANN by building an ANN with FA in hidden layer model, the building and training time has been reduced by $75 \%$ of the original model with keeping on the classification accuracy.

5. Applied FA with PCA method on multi-dimensional database with many different data types before ANN model Building and merging the results of FA in the hidden layer will contribute in reducing the ANN complexity degree then improving in building time and classification accuracy.

\section{REFERENCES}

[1] Mccllochw S, Pitts W, 1943, "A logical calculus of the ideas immanent in nervous activity". Bull Math Biophys 10(5):115-133

[2] P. Jeatrakul, K.W. Wong, 2009. "Comparing the Performance of Different Neural Networks for Binary Classification Problems". IEEE 
[3] B. Widrow, D. Rumelhard, and M. A. Lehr, 1994. "Neural networks: Applications in industry, business and science," Communications of the ACM, vol.37, pp. 93105.

[4] C. Charalambous, A. Charitou, and F. Kaourou, 2000, "Comparative analysis of artificial neural network models: application in bankruptcy prediction" in Neural Networks, IJCNN'99.International Joint Conference on Neural Networks, vol.6, pp. 3888-3893.

[5] L. Gang, B. Verma, and S. Kulkami, 2002. "Experimental analysis of neural network based feature extractors for cursive handwriting recognition," in Neural Networks. IJCNN '02. Proceedings of the 2002 International Joint Conference on Neural Networks, pp. 2837-2842.

[6] R. Rabuñal Juan, Dorado Julián, 2006. "Artificial Neural Networks in real life applications", Idea group publishing, London.

[7] Cheng H, Cai X, Min R, 2009."A novel approach to color normalization using neural network". Neural Computing and Applications 18(3), pp. 237-247.

[8] Rumelhart D, Hinton G, Williams R, 1986, "Learning representation by back-propagating errors". Nature.com vol. 323 , no. 6088 , pp. 533-538,

[9] Gupta Tarun Kumar, Raza Khalid, 2018. "Optimizing Deep Neural Network Architecture: A Tabu Search Based Approach". Computer Science, springer.

[10] Agah Arvin, 2013. "Medical Applications of Artificial Intelligence", CRC Press, Tylor \& Fracis Group. New York p. 204

[11] M. Gross, H. Luttermann, "Combining principal component analysis and neural networks for the recognition of human faces- a case study for man machine - communication, in: S.Y. Shin, T.L. Knuii (Eds.)",1993. Computer Graphics \& Applications, Proceedings of the Pacific Graphics, vol. 1, pp. 176-192.

[12] T.M. Khoshgoftaar, R.M. Szabo, 1996. "Using neural networks to predict software faults during testing", IEEE Transactions on Reliability, pp: 456-462.

[13] W.W. Hseih, B. Tang, 1998. "Applying neural network models to prediction and data analysis in meteorology and oceanography", Bulletin of the American Meteorological. Soc. 79 pp: $1855-1870$.

[14] O'Farrella, et al., 2005. "Combining principal component analysis with an artificial neural network to perform online quality assessment of food as it cooks in a largescale industrial oven", Sensors and Actuators B, Vol. 107, pp. 104-112.

[15] Bucinski, A., Baczek, T., Wasniewski, T., and Stefanowicz, M., 2005. "Clinical data analysis with the use of artificial neural networks (ANN) and principal component analysis (PCA) of patients with endometrial carcinoma", Reports on Practical Oncology and Radiotherapy, Vol. 10, pp. 239-248.

[16] Song J, Feng Y, 2006. "Hyperspectral data classification by independent component analysis and neural network". Remote sensing technology and application, pp. 115-119

[17] Zhang Y, 2007. "Artificial neural networks based on principal component analysis input selection for clinical pattern recognition analysis". Talanta 73(1) pp:68-75

[18] Gopi E, 2007. "Digital image forgery detection using artificial neural network and independent component analysis". Appl Math Comput 194(2) pp:540-543.

[19] Sousa, S.I.V. , Martins, F.G. , Alvim-Ferraz, M.C.M., Pereira, M.C., 2007. "Multiple linear regression and artificial neural networks based on principal components to predict ozone concentrations", Environmental Modelling \& Software, Vol. 22, pp. 97-103.

[20] Ravi, V., Pramodh, C., 2008. "Threshold accepting trained principal component neural network and feature subset selection: Application to bankruptcy prediction in banks", Applied Soft Computing, Vol. 8, pp. 1539-1548.

[21] Miguel Antonio, Llombart Jorge, Ortega Alfonso and Lleida Eduardo, 2017. "Tied Hidden Factors in Neural Networks for End-to-End Speaker Recognition", In Proc. Interspeech, pp 2819-2823, Stockholm, Sweden.

[22] Ding Shifei et al. et al., 2010. "Research of neural network algorithm based on factor analysis and cluster analysis", Neural Comput \& Applic (2011) 20:297-302 springer-Verlag London Limited.

[23] Zekić-Sušac Marijana et al., 2013. "Combining PCA analysis and artificial neural networks in modelling entrepreneurial intentions of students", Croatian Operational Research Review, Croatia, 4, P306-317.

[24] Taylor, Brian J., 2006. "Methods and Procedures for the Verification and Validation of Artificial Neural Networks", Springer Science \& Business Media.

[25] P. Arumugam, R. Ezhilarasi, 2017. "Data Mining based Neural Network Model for Rainfall Forecasting", International Journal of Computer Applications, Volume $170-$ No. 4

[26] Shafi Imran et al., 2006."Impact of Varying Neurons and Hidden Layers in Neural Network Architecture for a Time Frequency Application", IEEE International Multitopic Conference.

[27] Mehrotra K., Mohan C., Ranka S., 1997. "Element of Artificial Neural Networks", MIT Press, USA

[28] S. Haykin, 2008. "Neural networks and learning machines", third edition, Prentice Hall.

[29] R. Rojas, 1996. "Neural networks - a systematic introduction", Springer-Verlag.

[30] Dang Tuan Linh, Hoshino Yukinobu, 2019. "Improved PSO Algorithm for Training of Neural Network in Codesign Architecture", International Journal of Computer Applications, Volume 182 - No.44.

[31] Leskovec Jure, Rajaraman Anand, Ullman Jeffrey David, 2020. "Mining of Massive Datasets", Third edition, Cambridge University Press, pp. 517-520.

[32] Heaton Jeff, 2011. "Introduction to the Math of Neural Networks", Heaton Research, Inc., USA.

[33] Berrar Daniel, 2018. "Cross-validation", Encyclopedia of Bioinformatics and Computational Biology, Volume 1, Elsevier, pp. 542-545.

[34] Kamarthi, S. V., \& Pittner, S. 1999. "Accelerating neural network training using weight extrapolations". Neural Networks, 12, 1285-1299. 
[35] Du Ke-Lin, Swamy M. N. S., 2019. "Neural Networks and Statistical Learning", Springer-Verlag London, UK.

[36] Hagan Martin, Demuth Howard, Beale Mark, De Jesús Orlando, "Neural Network Design", Oklahoma State University, Stillwater, OK, United States, 2nd Edition, P13-1.

[37] Johnson Richard, Wichern Dean, 1998. "Applied Multivariate Statistical Analysis", fourth edition, prentice hall, Upper Saddle River, New jersey 07458.

[38] J,Salkind Neil, 2010. "Encyclopedia of Research
Design", 1st Edition, Kindle Edition, SAGE Publications, pp. 460-466.

[39] Saurabh Karsoliya, 2012. "Approximating Number of Hidden layer neurons in Multiple Hidden Layer BPNN Architecture" International Journal of Engineering Trends and Technology- Volume 3 Issue 6.

[40] S. Moro, P. Cortez and P. Rita. A Data-Driven Approach to Predict the Success of Bank Telemarketing. Decision Support Systems, In press, http://dx.doi.org/10.1016/j.dss.2014.03.01 